\title{
Improved difficult airway documentation using structured notes in Anesthesia Information Management Systems
}

\author{
Clyde Matava, MBCHB, MMed, MHSC (1) • Monica Caldeira-Kulbakas, PhD • \\ Jesse Chisholm, MBCHB
}

Received: 21 October 2019/Revised: 17 November 2019/Accepted: 18 November 2019/Published online: 26 November 2019

(C) Canadian Anesthesiologists' Society 2019

To the Editor,

Difficult airway management remains a challenge and each encounter requires accurate and quality documentation. ${ }^{1}$ Failure to document difficult airway may threaten patient safety during subsequent encounters. Complete documentation enables subsequent healthcare providers to plan approaches that avoid and minimize morbidity and mortality. ${ }^{2}$ Our institution recently transitioned from a paper anesthesia record system to an Anesthesia Information Management System (AIMS) as part of the enterprise electronic medical record solution, Epic (Epic Systems Corporation, Epic Anesthesia Module, Verona, WI, USA).

During the transition from paper to the AIMS record, we elected to use the structured format for a difficult airway note provided by the vendor. Our goal was to determine if note quality and completeness improved with the use of the AIMS structured difficult airway note compared with paper records. Approval was obtained (December 13, 2018) from our institutional quality and risk management for this retrospective chart audit. Following a literature review, local airway experts participated in a nominal group technique to develop the Toronto Difficult Airway

C. Matava, MBCHB, MMed, MHSC ( $\square)$.

J. Chisholm, MBCHB

Department of Anesthesia and Pain Medicine, The Hospital for Sick Children, Toronto, ON, Canada

e-mail: clyde.matava@sickkids.ca

Department of Anesthesia, Faculty of Medicine, University of Toronto, Toronto, ON, Canada

M. Caldeira-Kulbakas, $\mathrm{PhD}$

Department of Anesthesia and Pain Medicine, The Hospital for Sick Children, Toronto, ON, Canada
Documentation (TODAD) checklist, which comprises 18 items that assess the completeness of a difficult airway note (Figure A). Each item on the checklist accounts for one point of a possible 18-point score. To determine what constitutes a high-quality note, we used the e-CRABEL (CRAwford - BEresford - Lafferty) approach. ${ }^{3}$ The CRABEL score is reproducible and easy to use in objective assessments of medical record keeping quality. High-quality notes have previously been defined to have $85 \%$ of items in a checklist completed. ${ }^{3-5}$ Our primary outcome was the proportion of AIMS difficult airway notes scoring at least $85 \%$ (high quality) on the TODAD, compared with the legacy paper records. ${ }^{5}$

Based on a previous study on structured reporting, we determined $a$ priori that a sample size of 80 charts would detect a $50 \%$ improvement in the proportion of charts scoring $85 \%$ of TODAD. ${ }^{4} \mathrm{We}$ assumed a baseline proportion of $50 \%$ and an effect size of $50 \%$. Study data were collected using REDCap hosted at our institution. JMP ${ }^{\circledR}(\mathrm{JMP} \AA$, version 14, SAS Institute Inc., Cary, NC, USA 1989-2019) was used for analysis where a two-tailed $P<0.05$ was considered significant.

From the legacy paper records, we retrospectively accessed the last 40 consecutive difficult airway notes for patients undergoing non-airway surgery, who were intubated and flagged as "difficult airway". We also retrospectively accessed the first 40 consecutive structured difficult airway notes from the AIMS. Two investigators independently reviewed and scored each note using the TODAD checklist with any disagreement resolved by consensus.

The proportion of difficult airway notes with a TODAD score of $85 \%$ was $13 \%$ of the paper records vs $60 \%$ of the structured AIMS notes (relative risk, 12.5; 95\% confidence interval $[\mathrm{CI}], 3.2$ to $49.2 ; P<0.001$ ) (Figure $\mathrm{B}$ ). The mean 
Figure A) The Toronto Difficult Airway Documentation (TODAD) checklist. B) Boxwhisker plots depicting higher TODAD scores for Anesthesia Information Management System structured difficult airway notes compared with the paper record TODAD scores.
A

\begin{tabular}{|c|c|c|}
\hline \multicolumn{3}{|c|}{ Toronto Difficult Airway Documentation (TODAD) Checklist } \\
\hline Identifies time? & $\square$ & \\
\hline Identifies date? & $\square$ & \\
\hline Identifies procedure being performed? & $\square$ & \\
\hline Patient appropriate airway examination documented? & $\square$ & \\
\hline Discrete features predicting difficulty documented? & $\square$ & \\
\hline Mask ventilation attempt documented & $\square$ & \\
\hline Mask ventilation difficulty documented & $\square$ & \\
\hline Documents Cormack-Lehane view? & $\square$ & \\
\hline Documents equipment used for intubation & $\square$ & \\
\hline Documents number of intubation attempts? & $\square$ & \\
\hline Documents method of verifying intubation? & $\square$ & \\
\hline Documents type, size and depth of ETT? & $\square$ & \\
\hline Documents medications used? & $\square$ & \\
\hline Documents provider(s) that succeeded? & $\square$ & \\
\hline Identifies techniques that failed & $\square$ & \\
\hline Identifies other team members involved? & $\square$ & \\
\hline Documents complications or lack thereof & $\square$ & \\
\hline Documents recommendations for the future & $\square$ & \\
\hline Sub total & & \\
\hline Total & 18 & TODAD sc \\
\hline
\end{tabular}

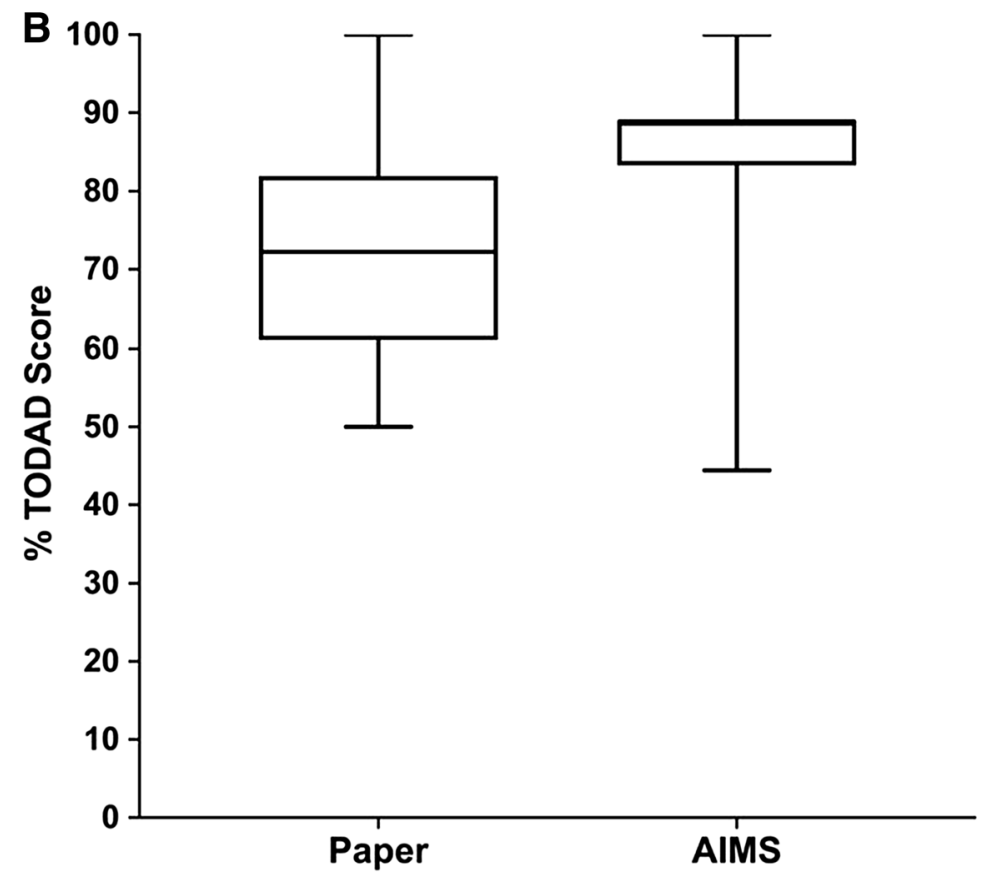

TODAD quality score increased from 71\% (95\% CI, 68 to 75) for paper records to $86 \%(95 \% \mathrm{CI}, 83$ to 89$)$ for structured AIMS notes $(P<0.001)$. A limitation of our study was that our department did not use paper-based structured difficult airway notes prior to adopting the AIMS. Nevertheless, our AIMS system provided the 
opportunity to use structured notes; we believed them to be superior.

In conclusion, we showed that using a structured AIMS difficult airway note improved the quality and completeness of difficult airway documentation. Institutions transitioning to AIMS should consider the use of structured, difficult airway notes. Further, the TODAD score may be used to monitor the quality and completeness of difficult airway documentation to ensure that vital information is available in the patient's electronic record.

Conflict of interest None.

Funding statement None.

Editorial responsibility This submission was handled by Dr. Hilary P. Grocott, Editor-in-Chief, Canadian Journal of Anesthesia.

\section{References}

1. Cook TM, MacDougall-Davis SR. Complications and failure of airway management. Br J Anaesth 2012; 109: i68-85.

2. Fiadjoe JE, Nishisaki A, Jagannathan $N$, et al. Airway management complications in children with difficult tracheal intubation from the Pediatric Difficult Intubation (PeDI) registry: a prospective cohort analysis. Lancet Respir Med 2016; 4: 37-48.

3. Crawford JR, Beresford TP, Lafferty KL. The CRABEL score-a method for auditing medical records. Ann R Coll Surg Engl 2001; 83: $65-8$.

4. Schöppe F, Sommer WH, Schmidutz F, et al. Structured reporting of X-rays for atraumatic shoulder pain: advantages over free text? BMC Med Imaging 2018; . https://doi.org/10.1186/s12880-0180262-8.

5. Myuran T, Turner O, Ben Doostdar B, Lovett B. The e-CRABEL score: an updated method for auditing medical records. BMJ Qual Improv Rep 2017; . https://doi.org/10.1136/bmjquality.u211253. w4529.

Publisher's Note Springer Nature remains neutral with regard to jurisdictional claims in published maps and institutional affiliations. 